This item was submitted to Loughborough's Institutional Repository (https://dspace.lboro.ac.uk/) by the author and is made available under the following Creative Commons Licence conditions.

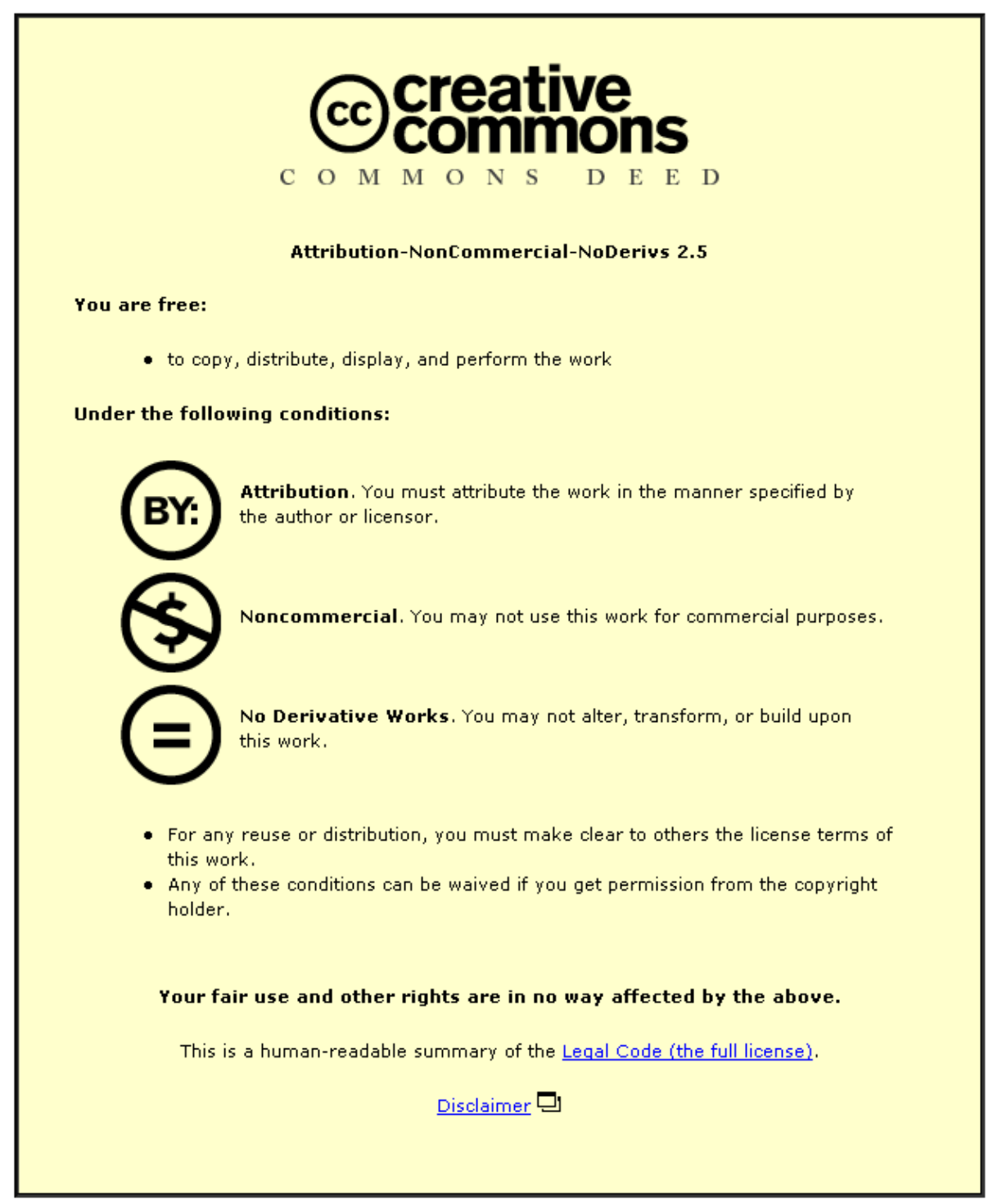

For the full text of this licence, please go to: http://creativecommons.org/licenses/by-nc-nd/2.5/ 


\title{
A MOBILE VIRTUAL ELECTROMAGNETICS LABORATORY FOR IPHONE
}

\author{
D. R. Browne ${ }^{\star}$, S. C. Pomeroy*, J. A. Flint ${ }^{\star}$ \\ *Dept. of Electronic and Electrical Engineering, Loughborough University, UK - Contact: D.R.Browne@lboro.ac.uk
}

Keywords: Electronic learning, Computational electromagnetics, Mobile computing, TLM.

\begin{abstract}
Modern mobile devices have now advanced to a point where they can execute computationally-intensive mathematical problems. The Transmission Line Matrix Modelling method (TLM) is a time-domain method allowing the computation of two and three dimensional electromagnetic fields and structures. TLM is able to model complex scenarios whilst utilising a computationally simple method to model structures in discrete spatial units. This paper presents an iPhone Application (App) containing a TLM solver. The simulation output is presented on the screen as the simulation progresses. The performance of the iPhone is sufficient to visualise full-field simulations and to provide an interactive interface for the user.
\end{abstract}

\section{Introduction}

Recent advances in modern mobile device technology and reductions in the costs to consumers have meant that "Smartphones"1 are becoming increasing popular, and comprise of 1 in 4 of mobile telephones in use [1]. Recently, they have reached a point where they can now be used in ways previously not possible, such as rendering 3D graphics for high-end video games.

With the increase in computation comes the possibility of mobile computational electromagnetics (mCEM). The i use of a Smartphone App for electromagnetic calculations has been described by Champagne [2], however to date only simple numerical and analytical methods have been applied. These devices are becoming capable of both solving and visualising fullfield problems and this paper describes development of an mCEM application for iPhone, using the Apple development environment XCode to develop and deploy a software package, or "App" for iPhone and iPod touch devices. The iPhone was selected due to its market penetration and its relatively high computational power [1]. However, other Smartphone devices are available and there is no reason why the approach taken in this paper could not be more widely applied.

\footnotetext{
1 Smartphones are technologically-advanced mobile phones that possess features such as a powerful processor, touch-screen interface, camera and GPS connectivity.
}

Advances in hardware now allow Smartphones to execute complex software. The iPhone is an example of a Smartphone that has sufficient performance in this regard. For example, the iPhone 4, contains a customdesigned System on Chip (SoC) consisting of an ARM Cortex-A8 CPU and a PowerVR SGX 535 Graphics Processing Unit (GPU). The A8 is capable of running at up to $1 \mathrm{GHz}$, and the SGX 535 supports advanced graphics rendering features as well as the high-end graphics library OpenGL 2.0. There is 512MB of onboard RAM, allowing a large amount of data to be cached during the execution of an application. This combination of features makes the iPhone an ideal candidate. Using Smartphones such as the iPhone allows data processing to now be moved onto the device itself, reducing infrastructure complexity by removing the need for separate workstations to complete data processing tasks.

\section{Introduction to TLM}

Two-dimensional TLM using shunt nodes provides a computationally simple method for simulating complex wave propagation and boundary interactions in a $2 \mathrm{D}$ plane [3] [4]. For the application presented in this paper, the touch-screen of the device is the plane to which the TLM simulation data is mapped, and 3D structures such as waveguides are represented in a top-down fashion on the device screen. The simple nature of the Scatter and Connect process that is the foundation of TLM allows all field components to be simultaneously available at each time step. This process is briefly explained in Figure 1, where 1a) shows initial excitation of the node mesh, 1b) shows propagation, or "scattering" of the pulse after the first time-step, and 1c) shows the scattering in the second time-step.

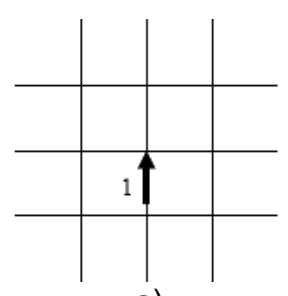

a)

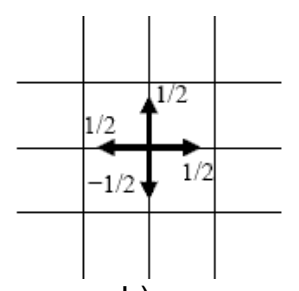

b)

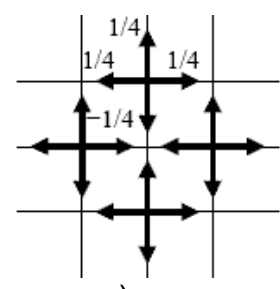

c)
Figure 1: Fundamentals of the 2D TLM process

\section{Application design}

The application is intended as a teaching aid for improving understanding of electromagnetics topics by visualising specific scenarios, and could be used as part 
of the taught section of an electromagnetics class. It is split into two user interface panels - the simulation view and the configuration view. This allows students to have the maximum amount of screen area available for interacting with simulations by keeping all in-depth configuration options on a separate panel. There are four modes accessible from the configuration panel (Gaussian Pulse, Sinusoidal Pulse, Virtual Ripple Tank and Waveguide). Examples of two of the simulation modes are shown in Figure 2. Wave intensity data is presented as a direct translation of the TLM node array intensity values mapped to a blue-to-red intensity colour spectrum. The image is scaled so that one TLM node corresponds to 4 pixels on screen The user has the ability to display the $\mathrm{E}$-field, $\mathrm{H}_{\mathrm{x}}$ or $\mathrm{H}_{\mathrm{y}}$ field components for all simulation modes, with the ability to change which field component is displayed during simulations.

Upon first loading, a virtual 'ripple tank' is displayed that responds to a user's touch. This allows the user to instantly interact with the "surface" and see the results of their manipulations. This flexibility was balanced with the "Waveguide" mode, which was specifically designed to present pre-configured phenomena, whilst still allowing the user the ability to change certain aspects of the simulation, such as wavelength.

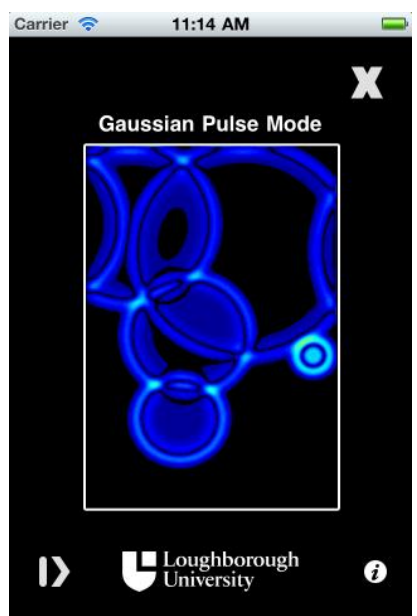

a)

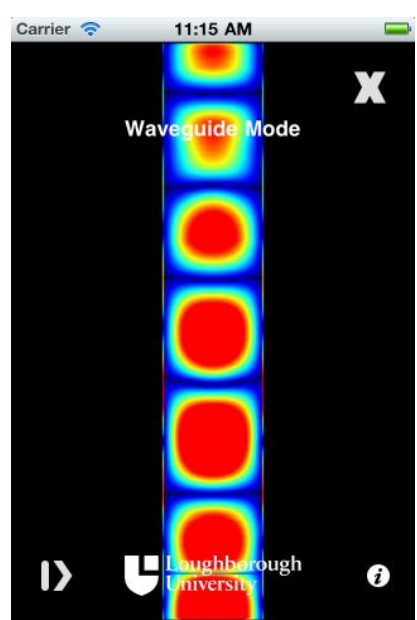

b)
Figure 2(a) the Virtual Ripple Tank and (b) Waveguide simulation modes

Table 1 shows the boundary conditions and mesh configurations corresponding to the modes shown previously in Figure 2, as well as the other simulation modes. The active node region corresponds to the area within the white rectangle in each image of Figure 2, and boundary condition types are applied to the edges of the region (Matched Termination Condition or Reflective). Whilst the reflective boundary has a coefficient of -1 , the MTC is as shown in Equation (1).

$$
M T C=\frac{\sqrt{2}-1}{\sqrt{2}+1}
$$

Individual mesh nodes use 16 bytes of RAM in the present implementation, allowing meshes larger than the screen dimensions to be stored. Testing has confirmed that the application is able to compute approximately 190,000 nodes/s. Table 1 details the total memory usage and maximum frame rates for each simulation mode.

\begin{tabular}{|c|c|c|c|c|}
\hline Mode & X Cond. & Y Cond. & RAM (KB) & Frames $/ \boldsymbol{s}$ \\
\hline Normal & -1 & -1 & 938 & 3.2 \\
Sinusoid & -1 & -1 & 938 & 3.2 \\
Ripple & -1 & -1 & 938 & 3.2 \\
W/guide & MTC & -1 & 600 & 4.9 \\
\hline
\end{tabular}

Table 1: Boundary conditions, RAM usage and frame rates for each simulation mode

Testing has demonstrated that the performance of the App differs between iPhone OS devices. Frame rates on an iPhone 3GS and iPhone 4 are approximately equivalent, whereas there is a noticeable increase in speed using the iPad. This is due to the fact that the Cortex-A8 CPU runs at $1 \mathrm{GHz}$ on the iPad, whereas it is under-clocked to preserve battery life in the iPhone 4 and $3 G S$.

\section{Conclusions and further work}

It has been demonstrated that it is possible to implement a virtual laboratory for simulating full-field electromagnetic phenomena using the iPhone as a platform, with TLM used as the computation method. Results show that it is possible for simulations to be executed at suitable speeds to demonstrate various types of electromagnetic phenomena to an end user as they are generated. The application is currently available for download from the Apple App Store [5]. Further work intends to improve the feature set of the software, and to explore its use in an educational context. Work currently in progress includes deployment to student cohorts to determine the pedagogical benefits of such an approach.

\section{References}

[1] D. Kellogg. "iPhone vs. Android", http://blog.nielsen.com/nielsenwire/online_mobile/ iphone-vs-android/.

[2] N. Champagne. "jEMLab for iPhone" (iPhone App), http://itunes.apple.com/gb/app/jemlab/id330716516 ? $\mathrm{mt}=8$.

[3] C. Christopoulos. "The Transmission Line Modelling Method", IEEE Press, pp. 90-98, (1995).

[4] P. B. Johns, R. L. Beurle. "Numerical Solution of 2Dimensional Scattering Problems using a Transmission-Line Matrix", Proc. IEE, 118, pp 1203-1208, (1971).

[5] D. R. Browne. "Loughborough Wave Lab" (iPhone App), http://itunes.apple.com/gb/app/loughboroughwave-lab/id402424695?mt=8. 